\title{
Probing meson spectral functions with double differential dilepton spectra in heavy-ion collisions
}

\author{
B. Kämpfer ${ }^{a}$, O.P. Pavlenko ${ }^{a, b}$ \\ ${ }^{a}$ Forschungszentrum Rossendorf, PF 510119, 01314 Dresden, Germany \\ ${ }^{b}$ Institute for Theoretical Physics, 252143 Kiev - 143, Ukraine
}

\begin{abstract}
The double differential dilepton spectrum $d N /\left(d M_{\perp}^{2} d M^{2}\right)$ at fixed transverse mass $M_{\perp}$ allows a direct access to the vector meson spectral functions. Within a fireball model the sensitivity of $d N /\left(d M_{\perp}^{2} d M^{2}\right)$ against variations of both the in-medium properties of mesons and the dynamics of the fireball is investigated. In contrast to the integrated invariant-mass spectrum $d N / d M^{2}$, in the spectrum $d N / d M^{2} d M_{\perp}^{2}$ with fixed $M_{\perp}$ the $\omega$ signal is clearly seen as bump riding on the $\rho$ background even in case of strong in-medium modifications.
\end{abstract}

Typeset using REVTEX 


\section{INTRODUCTION}

A strong motivation for the starting experiments with the HADES detector [1] at the heavy-ion synchrotron SIS in GSI Darmstadt is to search for direct evidences of in-medium modifications of hadrons via the dilepton $\left(e^{+} e^{-}\right)$channel. Such modifications, like the $\rho$ meson "melting" and possible in-medium mass shifts, being related to chiral symmetry restoration, are considered now as the main cause of the low-mass dilepton excess observed in heavy-ion experiments at CERN-SPS energies [2]. According to the present understanding of the chiral phase transition [3, 1 , the chiral condensate $\langle q \bar{q}\rangle$ as order parameter decreases with increasing temperature $T$ and baryon density $n$. This leads to modifications of the meson spectral functions since they are coupled to $\langle q \bar{q}\rangle$. While the dependence of the chiral condensate as a function of the temperature at small baryon density is quite smooth up to a critical temperature of $T_{c} \sim 170 \mathrm{MeV}$ [5], the dropping of $\langle q \bar{q}\rangle$ with increasing density at small temperature is more pronounced already at moderate densities. For instance, in the QCD sum rule analysis [6], which is in accordance with the anticipated Brown-Rho scaling [7], a density dependence of the hadron mass shift like $m^{*}=m\left(1-0.18 n / n_{0}\right)$ has been predicted, so that even at the nuclear saturation density $n=n_{0}=0.15 \mathrm{fm}^{-3}$ a noticeable change of the in-medium mass $m^{*}$ emerges. Unfortunately, in the case of heavy-ion collisions, where densities up to $3 n_{0}$ can be expected at SIS energies, the remarkable advantage of the strong density dependence meets a problem when trying to identify the predicted mass shift by the direct dilepton decay of vector mesons. Due to the collective expansion of the baryonic matter the density $n$ depends on time so that obviously a time average of the mass shift is to be expected, i.e. the mass shift effect is smeared out over an interval related to the change of $n$.

To extract the wanted information on the in-medium meson spectral function via dilepton measurements in heavy-ion collisions one has also to take into account the general properties of the dilepton spectra in a thermalized system which undergoes a collective expansion. For instance, in a dense system not too far from local thermal equilibrium, the dilepton rate is known to be governed mainly by the Boltzmann factor $\exp \{-E / T\}$, where $E=\sqrt{M^{2}+\vec{Q}^{2}}$ is the lepton pair energy, and the pair is characterized by the invariant mass $M$ and threemomentum $\vec{Q}$. That means the dilepton spectrum $d N / d M^{2}$ as a function of the invariant mass is a convolution of spectral functions and the Boltzmann factor. For temperatures $T<100 \mathrm{MeV}$, as estimated for SIS energies, and dilepton masses in the region of the light vector mesons, $M \sim m_{V} \sim 770 \mathrm{MeV}$, the ratio $M / T$ can be in the order of 10 and, as a consequence, the resulting spectra are very steep. This can make an identification of possible mass shifts, superimposed to (at least collision) broadening, rather difficult. Moreover, dynamical effects, caused by flow and cooling, can even stronger mask the wanted information.

In the present note we propose to extend the usual analysis of the integrated spectrum $d N / d M^{2}$ to the more informative double differential spectrum $d N /\left(d M_{\perp}^{2} d M^{2}\right)$, where $M_{\perp}=$ $\sqrt{M^{2}+Q_{\perp}^{2}}$ is the transverse mass of the pair with transverse two-momentum $\vec{Q}_{\perp}$. This allows to avoid the majority of the above mentioned difficulties in extracting the in-medium vector meson spectral function on the basis of dilepton measurements in heavy-ion collisions. In particular, by taking the transverse mass $M_{\perp}$ at a fixed value (or in a sufficiently narrow 
bin) one gets rid of the steep slope of the invariant mass spectrum as we show below. As a result the spectral function is seen more clearly, and possible in-medium effects become more pronounced.

To demonstrate the very idea of our approach we employ here a fireball model for describing the space-time evolution of the dense matter formed in central collisions of heavy nuclei at SIS energies. In spite of the schematic character of the model, it is useful to estimate various characteristics, such as the relative contributions of vector mesons decaying inside and outside of the fireball and Dalitz decays of mesons and the $\Delta$ as well, which are related to observables accessible in HADES experiments. Along with more complicated transport model calculations [8,9], we consider our approach as a first step towards elucidating the most appropriate observables for verifying in-medium changes of meson properties in heavy-ion collisions.

Our paper is organized as follows. In Sect. 2, we present the parameterizations of the dilepton rates which we use in our exploratory studies. The fireball dynamics is given in Sect. 3. The resulting dilepton spectra are discussed in Sect. 4, where we contrast the dileptons emitted during the fireball's lifetime and such dileptons which stem from hadron decays after freeze-out. The discussion can be found in sect. 5 together with remarks on the support of the presented results by calculations based on a BUU code.

\section{DILEPTON RATES}

The dilepton rate from vector meson decays, $V \rightarrow e^{+} e^{-}$with $V=\rho, \omega, \phi$, in an ideal resonance gas approximation is given by

$$
\frac{d N}{d^{4} x d^{4} Q}=\sum_{V} \frac{2 d_{V}}{(2 \pi)^{3}} \exp \left\{-\frac{u \cdot Q}{T}\right\} \frac{1}{\pi} \frac{\left(m_{V} \Gamma_{V}^{\text {tot }}\right)\left(M \Gamma_{V \rightarrow e^{+} e^{-}}\right)}{\left(M^{2}-m_{V}^{2}\right)^{2}+\left(m_{V} \Gamma_{V}^{\text {tot }}\right)^{2}},
$$

where $\Gamma_{V}^{\text {tot }}$ and $\Gamma_{V \rightarrow e^{+} e^{-}}$denote the total and dilepton decay widths, $m_{V}$ is the vector meson mass, and $d_{V}$ stands for the degeneracy factor. The lepton pair has the four-momentum $Q^{\mu}=\left(M_{\perp} \cosh Y, M_{\perp} \sinh Y, \vec{Q}_{\perp}\right)$, where $Y$ denotes the pair rapidity. In (1) we use the Breit-Wigner parameterization of the meson spectral function,

$$
A_{V}(m)=\frac{1}{\pi} \frac{m_{V} \Gamma_{V}^{\mathrm{tot}}}{\left(m^{2}-m_{V}^{2}\right)^{2}+\left(m_{V} \Gamma_{V}^{\mathrm{tot}}\right)^{2}},
$$

which can be thought to result from the generalization of an on-mass shell delta function to the case of an unstable particle [10]. Such an ansatz is also used for QCD sum rule analyzes 11] of vector meson properties in a nuclear medium. Due to four-momentum conservation one gets immediately $m^{2}=Q^{2} \equiv M^{2}$.

It should be emphasized that, within the vector dominance model, the dependences $\Gamma_{\rho \rightarrow e^{+} e^{-}} \propto M^{-2}$ and $\Gamma_{\rho}^{\text {tot }} \propto M^{2}$ just cancel, therefore, in (1) the widths are understood to be

constant. A remaining $M$ dependence stemming from $\Gamma_{\rho \rightarrow \pi^{+} \pi^{-}}^{\text {tot }} \propto\left(1-\frac{4 m_{\pi}^{2}}{M^{2}}\right)^{3 / 2}$ is partially mimicked by the factor $M$ in the numerator in (1). We have checked that the rate described by (11) agrees with the standard parameterization [10] of the process $\pi^{+} \pi^{-} \rightarrow e^{+} e^{-}$in the 
intervals $400 \mathrm{MeVH}<M<1000 \mathrm{MeV}$ and $50 \mathrm{MeV}<T<100 \mathrm{MeV}$. For the narrow $\omega$ and $\phi$ mesons these subtleties are not important.

The collective expansion of the matter is characterized by the four-velocity $u^{\mu}(x)$ depending generally on space and time, as also does the temperature $T(x)$. In spite of the approximative character of (1) it is useful for demonstrating how the in-medium modifications in the spectral function (2) show up in the dilepton spectra if flow effects of the matter are taken into account.

Integrating the rate (1) over the three-momentum of the lepton pair one gets the rate as a function of the invariant mass,

$$
\frac{d N}{d^{4} x d M^{2}}=\sum_{V} \frac{d_{V}}{2 \pi^{3}} M T K_{1}\left(\frac{M}{T}\right) \frac{\left(m_{V} \Gamma_{V}^{\mathrm{tot}}\right)\left(M \Gamma_{V \rightarrow e^{+} e^{-}}\right)}{\left(M^{2}-m_{V}^{2}\right)^{2}+\left(m_{V} \Gamma_{V}^{\mathrm{tot}}\right)^{2}},
$$

where $K_{1}$ is a Bessel functions.

In Fig. 1a we plot the rate (3) at a temperature of $T=70 \mathrm{MeV}$ to demonstrate the general tendency of the very steeply dropping spectrum with increasing $M$. Since we do not advocate in the present note any particular functional form of the meson spectral function (2), which is under intense debate so far, we parameterize schematically here the in-medium width broadening by the change of the total vacuum width according to $\Gamma_{V} \rightarrow \Gamma_{V}^{*}=b \Gamma_{V}$ with a constant parameter $\mathrm{b}=3$ for all vector mesons. (The position of the in-medium vector meson peak is not important for this example.) As seen in Fig. 1a the important effect of the vector meson broadening is smeared out by the rapidly dropping thermal weight factor $K_{1}(M / T)$ in (3). No peak related to the $\rho$ meson remains. The $\omega$ peak is still visible, but not very pronounced. The strongest peak structure is related to the $\phi$ meson.

The above example can be contrasted to the double differential rate $d N /\left(d^{4} x d M_{\perp}^{2} d M^{2}\right)$ obtained from (看) by integrating over the dilepton rapidity and the polar angle. For a spherically symmetric source we find

$$
\frac{d N}{d^{4} x d M_{\perp}^{2} d M^{2}}=\sum_{V} \frac{2 d_{V}}{(2 \pi)^{3}} K_{0}\left(\frac{M_{\perp} \gamma}{T}\right) I_{0}\left(\frac{Q_{\perp} v_{r} \gamma}{T}\right) \frac{\left(m_{V} \Gamma_{V}^{\mathrm{tot}}\right)\left(M \Gamma_{V \rightarrow e^{+} e^{-}}\right)}{\left(M^{2}-m_{V}^{2}\right)^{2}+\left(m_{V} \Gamma_{V}^{\mathrm{tot}}\right)^{2}}
$$

where $\gamma=1 / \sqrt{1-v_{r}^{2}}$ is the Lorentz factor corresponding to the radial flow three-velocity $v_{r}$, and $Q_{\perp}=\sqrt{M_{\perp}^{2}-M^{2}}$. One can infer from (4) that, by fixing the transverse mass, the thermal weight is frozen in. For sufficiently small values of the flow velocity $v_{r}$ the rate $d N /\left(d^{4} x d M_{\perp}^{2} d M^{2}\right)$ as function of the invariant mass at fixed $M_{\perp}$ reflects directly the shape of the meson spectral function. In Fig. 1b we show this remarkable property for $M_{\perp}=1.3$ $\mathrm{GeV}, v_{r}=0.3$ and $T=70 \mathrm{MeV}$. The $\rho$ bump is here clearly visible below the $\omega$ peak; the $\phi$ needle is more pronounced. The price is, of course, a diminished count rate. However, for

\footnotetext{
${ }^{1}$ At this and smaller values of $M$ the rate is anyhow dominated by Dalitz decays. Therefore, we do not attempt a modification of the low- $M$ tail of (1) which arises from a particular model. For more involved expressions of the rate we refer the interested reader, e.g., to [12], where also the coupling to the baryon charge is included.
} 
$M_{\perp}=0.9 \mathrm{GeV}$ the rate is still not too much smaller in comparison with the rate displayed in in Fig. 1a at $M=0.9 \mathrm{GeV}$.

In Fig. $1 \mathrm{~b}$ we show that the rate increases in the $\rho$ region with increasing values of the radial velocity $v_{r}$. This fact is known since some time [13] as enhancement of the $\rho$ peak due to transverse flow. Note that the rate $d N /\left(d^{4} x d M^{2}\right)$ is completely unaffected by flow effects.

It should be stressed that other representations of the double differential rate, such as $d N / d^{4} x d Q_{\perp}^{2} d M^{2}$, are less suitable for an access to the spectral function.

\section{DYNAMICS}

To obtain the dilepton spectrum from the meson decay rate (11) one needs to specify the space-time evolution of the matter. According to our experience [14] the detailed knowledge of the space-time evolution is not necessary to describe the experimental dilepton spectra in relativistic heavy-ion collisions in the low-mass region, where the in-medium modifications of the meson spectral function play a crucial role. In this line, and also to avoid too many parameters, we employ here a variant of the blast wave model [15] with constant radial expansion velocity $v_{r}$. In such a model the radius of the fireball increases like $R(t)=$ $R_{i}+v_{r} t$. From the analysis of hadron ratios in heavy-ion reactions at SIS energies a freezeout temperature of $T_{f}=50 \mathrm{MeV}$ at freeze-out baryon density $n_{f}=0.3 n_{0}$ is obtained [16]. Extrapolating back along an isentropic line by using a resonance gas model as in [16] up to $n_{i}=3 n_{0}$ (as suggested by transport code simulations for the maximum density at SIS energies) one finds $T_{i}=90 \mathrm{MeV}$. The freeze-out time $t_{f}$ is determined by baryon conservation within the fireball volume; we use as baryon participant number $N=330$. The time evolution starts at initial time $t_{i}=0$. We take $v_{r}=0.3$ as a reasonable average according to [16]. By these parameters the dynamics of the fireball is completely fixed.

\section{DILEPTON SPECTRA}

\section{A. The fireball radiation}

Since the aim of our work is a demonstration of the advantage of the double differential dilepton spectrum $d N /\left(d M_{\perp}^{2} d M^{2}\right)$ independently of a given form of the vector meson's spectral function $A_{V}$, we do not use specific functional changes of $A_{V}$ but consider a variety of possible parameterizations of the in-medium effects via mass shifts and width broadenings, i.e., changes of the parameters $\Gamma_{V}^{\text {tot }}$ and $m_{V}$. As long as the quantitative description of $A_{V}(m)$ in a medium is matter of debate [2] such a procedure seems appropriate.

We begin with an extreme case where the $\rho$ meson mass shift is $\Delta m_{\rho}=300 \mathrm{MeV}$, while the $\omega$ meson is unaffected, i.e. $\Delta m_{\omega}=0$, and $m_{V}^{*}=m_{V}-\Delta m_{V}$. The in-medium widths of both the low-lying vector mesons is increased by $b=3$. The resulting spectra are displayed in Fig. 2, and we have chosen $M_{\perp}=0.9 \mathrm{GeV}$. In contrast to the integrated rate $d N / d M^{2}$, where the $\rho$ peak disappears (cf. Fig. 2a), one can clearly observe the splitting of both vector meson peaks in the $d N /\left(d M_{\perp}^{2} d M^{2}\right)$ spectrum (cf. Fig. $2 \mathrm{~b}$ ), thus reflecting the

original modifications of the $\rho, \omega$ spectral functions. 
Another similarly extreme case is to keep the $\rho$ mass fixed and to shift the $\omega$ mass by $\Delta m_{\omega}=300 \mathrm{MeV}$ down; again with widths broadening factor $b=3$. As seen in Fig. 3 the information on the change of the meson properties is best visible in the $d N /\left(d M_{\perp}^{2} d M^{2}\right)$ spectrum, where a strong $\omega$ peak and some part of the $\rho$ bump are distinguishable (cf. Fig $3 \mathrm{~b}$ ). Otherwise, the shifted $\omega$ peak is also nicely visible in the $d N / d M^{2}$ spectrum (cf. Fig. 3a) because it "rides" on the $\rho$ background and becomes more pronounced; a $\rho$ bump is here again hardly visible.

In more realistic considerations the values of the meson mass shifts and width broadenings should depend on the baryon density and temperature which are strongly time dependent. This in turn causes an additional smearing of the peaks of the spectral functions. To demonstrate that $d N /\left(d M_{\perp}^{2} d M^{2}\right)$ is still useful to elucidate occurrence of in-medium modifications we parameterize now the in-medium $\omega$ mass by $m_{\omega}^{*}=m_{\omega}\left(1-\delta_{\omega} \cdot n / n_{0}\right)$, $\Gamma_{\omega}^{\text {tot* }}=\Gamma_{\omega}^{\text {tot }}+\delta \Gamma_{\omega} n / n_{0}$ with $\delta_{\omega}=0.08$ and $\delta \Gamma_{\omega}=20 \mathrm{MeV}$, and for the $\rho$ meson we assume only a broadening according to $\Gamma_{\rho}^{\mathrm{tot} *}=\Gamma_{\rho}^{\mathrm{tot}}+\delta \Gamma_{\rho} \cdot n / n_{0}$ with $\delta \Gamma_{\rho}=400 \mathrm{MeV}$, where the superscript $*$ denotes again the in-medium quantities. These parameterizations are in line with current expectations [17] that the $\rho$ meson suffers a very strong in-medium broadening, while for the $\omega$ meson one can expect moderate modifications. The resulting spectra are displayed in Fig. 4. In contrast to the integrated invariant mass spectrum $d N / d M^{2}$ (cf. Fig. 4a), in the spectrum $d N /\left(d M_{\perp}^{2} d M^{2}\right)$ with fixed $M_{\perp}$ (cf. Fig. $\left.4 \mathrm{~b}\right)$ the $\omega$ meson contribution is clearly seen as shifted bump riding on the $\rho$ background, in spite of the smearing caused by the space time evolution. (Notice that in Fig. 4a the smeared $\omega$ contribution could easily be misinterpreted as $\rho$ bump.) Therefore, the measurement of the double differential dilepton spectrum at fixed $M_{\perp}$ offers much better chances to identify a possible $\omega$ mass shift.

It should be stressed that changes of $T_{i}$ cause a noticeable up/down shift of the spectra but let the shapes nearly the same.

\section{B. Background contributions}

Within the given model one can also estimate various background contributions. For instance, the contributions of vector meson decays after freeze-out can be consistently evaluated. The invariant mass spectrum of such decays can be calculated from

$$
\frac{d N}{d M^{2}}=\sum_{V} \frac{1}{\Gamma_{V}^{\mathrm{tot}}} \frac{d \Gamma_{V \rightarrow e^{+} e^{-}}}{d M^{2}} N_{V}\left(T_{f}, m_{V}=M\right),
$$

where $d \Gamma_{V \rightarrow e^{+} e^{-}} / d M^{2}=\Gamma_{V \rightarrow e^{+} e^{-}} A_{V}\left(M^{2}\right)$ and $N_{V}\left(T_{f}, m_{V}\right)$ is the number of vector mesons at freeze-out. This number is calculable within the Cooper-Frye formalism [18, which includes relativistic effects related to the matter's expansion. In our spherical fireball model with constant radial flow velocity one gets

$$
\begin{aligned}
N_{V}\left(T_{f}, m_{V}\right) & =\frac{d_{V}}{(2 \pi)^{3}} \frac{4 \pi R_{f}^{3}}{3 \gamma} \int d m^{2} \frac{m_{V} \Gamma_{V}^{\mathrm{tot}}}{\left(m^{2}-m_{V}^{2}\right)^{2}+\left(m_{V} \Gamma_{V}^{\mathrm{tot}}\right)^{2}} \\
& \times \int d p_{\perp}^{2} \sqrt{\frac{2 \pi T_{f}}{\gamma m_{\perp}}} \exp \left\{-\frac{m_{\perp} \gamma}{T_{f}}\right\}\left[\frac{\sinh a_{f}}{a_{f}}\left(\gamma p_{\perp}+T_{f}\right)-T_{f} \cosh a_{f}\right],
\end{aligned}
$$


where $m_{\perp}=\sqrt{m^{2}+p_{\perp}^{2}}$ is the transverse mass of the meson $V$ with transverse momentum $p_{\perp}$, and $a_{f}=\gamma v_{r} p_{\perp} / T_{f}$. In deriving (6) we employ the meson spectral function $A_{V}(m)$ from (2).

The calculations show that even for the "long-living" $\omega, \phi$ mesons the decay contribution after freeze-out is considerably smaller than the decay contribution during the fireball's life time. This is in contrast to naive expectations based on simple life time arguments, according to which the life time of the $\omega$ meson is $23 \mathrm{fm} / \mathrm{c}$ compared to the life time of the thermalized system $\mathcal{O}(10) \mathrm{fm} / \mathrm{c}$. To arrive at a consistent estimate one has to take into account the evolution and the hypothesis of thermalization. The latter assumption is the key to understand the small contribution of the decays after freeze-out: In the early stage the temperature is much higher and, according to the assumed chemical equilibrium, the appearance of vector mesons is much more frequent than at freeze-out. At the pole positions the ratio of the fireball to the freeze-out decay contributions can be roughly estimated by $\exp \left\{-m_{V} / T_{i}\right\} / \exp \left\{-m_{V} / T_{f}\right\}$, because of the dominating exponential factors.

It should be emphasized that the analysis of the hadron multiplicities at freeze-out in [16] supports the chemical equilibrium picture for some hadrons. In the present work we assume chemical equilibrium for the full time evolution. This equilibrium is certainly not maintained during the time evolution for all hadron species. For instance, the $\omega$ production via the inverse decay reaction $3 \pi \rightarrow \omega$ is rather unlikely. Such effects can be simulated by additional chemical off-equilibrium potentials, which keep certain hadron species on a higher number than we have assumed here. In this respect our estimates, e.g., of the $\omega$ numbers, serve as unfavorable lower limits when assuming particle production in the stages of highest temperature and density.

While we focus on the direct decay channels $V \rightarrow e^{+} e^{-}$, the Dalitz decay channels, like $\Delta \rightarrow N e^{+} e^{-}, \phi \rightarrow \eta e^{+} e^{-}, \omega \rightarrow \pi^{0} e^{+} e^{-}, \eta^{\prime} \rightarrow \pi^{0}(\eta) e^{+} e^{-}, \eta \rightarrow \gamma e^{+} e^{-}, \pi_{0} \rightarrow \gamma e^{+} e^{-}$etc. populate the invariant mass spectrum in the low-mass region (for a recent comprehensive analysis of the various other channels cf. [19]). These Dalitz decays are calculated by $(5,6)$ with $d \Gamma_{V \rightarrow e^{+} e^{-}} / d M^{2}$ to be replaced by $d \Gamma_{A \rightarrow B e^{+} e^{-}} / d M^{2}$ (for hadron decays after freeze-out) and

$$
\begin{aligned}
\frac{d N_{A \rightarrow B e^{+} e^{-}}}{d^{4} x d M^{2}} & =\frac{d_{A}}{2 \pi^{3}} \int d m m^{2} \frac{m_{A} \Gamma_{A}^{\mathrm{tot}}}{\left(m^{2}-m_{A}^{2}\right)^{2}+\left(m_{A} \Gamma_{A}^{\mathrm{tot}}\right)^{2}} \frac{d \Gamma_{A \rightarrow B e^{+} e^{-}}}{d M^{2}} T K_{1}\left(\frac{m}{T}\right), \\
\frac{d \Gamma_{A \rightarrow B e^{+} e^{-}}}{d M^{2}} & =\frac{\alpha}{3 \pi M^{2}} \Gamma_{A \rightarrow B \gamma^{*}},
\end{aligned}
$$

(for hadron decays during the fireball's life time ), where $\Gamma_{A \rightarrow B \gamma^{*}}$ is taken from 10 for meson decays and from [9] for $\Delta$ decays.

With respect to the $\Delta$ Dalitz contribution we mention that the number of $\Delta$ resonances depends on the baryo-chemical potential. The latter one is in our fireball model approximately $850 \mathrm{MeV}$ and fairly well time independent. The quoted value results from the chemical freeze-out analysis in [16], and the approximate time independence is a special feature of the isentropic line with specific entropy of 5 in the interval $n=0.3 \cdots 3 n_{0}$.

We have checked that the sum of these Dalitz decay channels at $M>0.5 \mathrm{GeV}$ is below the direct vector meson decay contributions (see also remarks in the next section). 


\section{DISCUSSION AND SUMMARY}

In summary we have demonstrated that, within a fireball model and a simple parameterization of the rate, the double differential dilepton spectra $d N /\left(d M_{\perp}^{2} d M^{2}\right)$ at fixed value of $M_{\perp}$ can deliver valuable information on a possible change of the in-medium properties of vector mesons. The usually considered invariant mass spectrum $d N / d M^{2}$ contains a convolution of the spectral function with a thermal weighting function which is too strong such that modifications of the spectral functions are difficult to disentangle, in particular if the dynamics of the system causes an additional smearing.

We consider our fireball model as first step towards finding the most appropriate characteristics of dilepton spectra to probe in-medium vector meson spectral functions. The results obtained, in particular the $d N / d M^{2} d M_{\perp}^{2}$ spectra, should be confirmed by more realistic transport calculations. Indeed, using the BUU code developed from 20 we performed calculations in line with the present work. These calculations show very clearly that, when selecting the interval $850 \mathrm{MeV}<M_{\perp}<950 \mathrm{MeV}$, (i) the resulting spectrum $d N / d M^{2} d M_{\perp}^{2}$ is dominated by the channel $\pi^{+} \pi^{-} \rightarrow \rho \rightarrow e^{+} e^{-}$in the interval $300 \mathrm{MeV}<M<850 \mathrm{MeV}$ thus directly reflecting the shape of the $\rho$ formfactor, (ii) in the high- $M$ tail also direct $\rho$ decays, with $\rho$ 's stemming from other channels than $\pi^{+} \pi^{-}$annihilations, can become as large as the $\pi^{+} \pi^{-}$annihilation contribution, (iii) the $\omega$ rides on the $\rho$ bump, and (iv) the $p n$ bremsstrahlung, $\Delta$ Dalitz and $\eta$ Dalitz decays are negligible in the mentioned $M$ interval. The details of these BUU calculations will be presented elsewhere [21].

To make reliable predictions for the future experiments at HADES one needs also additional analyses of the mass shifts and widths with respect to both the baryon density and temperature dependences in the regions expected to be achieved in heavy-ion collisions at SIS.

Acknowledgments: We thank M. Gorenstein, R. Rapp, A. Sibirtsev, Gy. Wolf and G. Zinovjev for stimulating discussions. O.P.P. thanks for the warm hospitality of the nuclear theory group in the Research Center Rossendorf. The work is supported by BMBF grant 06DR829/1, WTZ UKR-008/98, and STCU-015. 


\section{REFERENCES}

[1] J. Friese (for the HADES collaboration), Prog. Part. Nucl. Phys. 42, 235 (1999)

[2] R. Rapp, Nucl. Phys. A 661, 33c (1999);

R. Rapp, J. Wambach, hep-ph/9909229, Adv. Nucl. Phys. in print

[3] G. Brown, M. Rho, Phys. Rep. 269, 333 (1996)

[4] R.D. Pisarski, hep-ph/9503330, unpublished

[5] F. Karsch, Nucl. Phys. A 590, 367c (1995)

[6] T. Hatsuda, S.H. Lee, Phys. Rev. C 46, R34 (1992)

[7] G. Brown, M. Rho, Phys. Rev. Lett. 66, 2720 (1991);

G. Brown, G.Q. Li, R. Rapp, M. Rho, J. Wambach, Acta Phys. Pol. B 29, 2309 (1998)

[8] W. Cassing, E.L. Bratkovskaya, Phys. Rep. 308, 65 (1999)

[9] C. Ernst, S.A. Bass, M. Belkacem, H. Stöcker, W. Greiner, Phys. Rev. C 58, 447 (1998)

[10] P. Koch, Z. Phys. C 57, 283 (1993);

C.M. Hung, E.V. Shuryak, Phys. Rev. C 56, 453 (1997)

[11] S. Leupold, W. Peters, U. Mosel, Nucl. Phys. A 628, 311 (1998)

[12] O. Teodorescu, A.K. Dutt-Mazumder, C. Gale, Phys. Rev. C 61, 051901 (2000) and nucl-th/0008006

[13] K. Kajantie, M. Kataja, L. McLerran, P.V. Ruuskanen, Phys. Rev. D 34, 811 (1986)

[14] K. Gallmeister, B. Kämpfer, O.P. Pavlenko, Phys. Lett. B 473, 20 (2000);

K. Gallmeister, B. Kämpfer, O.P. Pavlenko, Phys. Rev. C 62, 057901 (2000);

K. Gallmeister, B. Kämpfer, O.P. Pavlenko, C. Gale, hep-ph/0010332

[15] P.J. Simson, I.O. Rasmussen, Phys. Rev. Lett. 42, 880 (1979)

[16] J. Cleymans, H. Oeschler, K. Redlich, Phys. Rev. C 59, 1663 (1999)

[17] F. Klingl, N. Kaiser, W. Weise, Nucl. Phys. A 624, 527 (1997)

[18] E. Cooper, G. Frye, Phys. Rev. D 10, 186 (1974)

[19] A. Faessler, C. Fuchs, M.I. Krivoruchenko, Phys. Rev. C 61, 035206 (2000)

[20] Gy. Wolf, G. Batko, W. Cassing, U. Mosel, K. Niita, M. Schäfer, Nucl Phys. A 517, 615 (1990);

Gy. Wolf, B. Friman, M. Soyer, Nucl. Phys. A 640, 129 (1998)

[21] B. Kämpfer, O.P. Pavlenko, Gy. Wolf, in preparation 


\section{FIGURES}
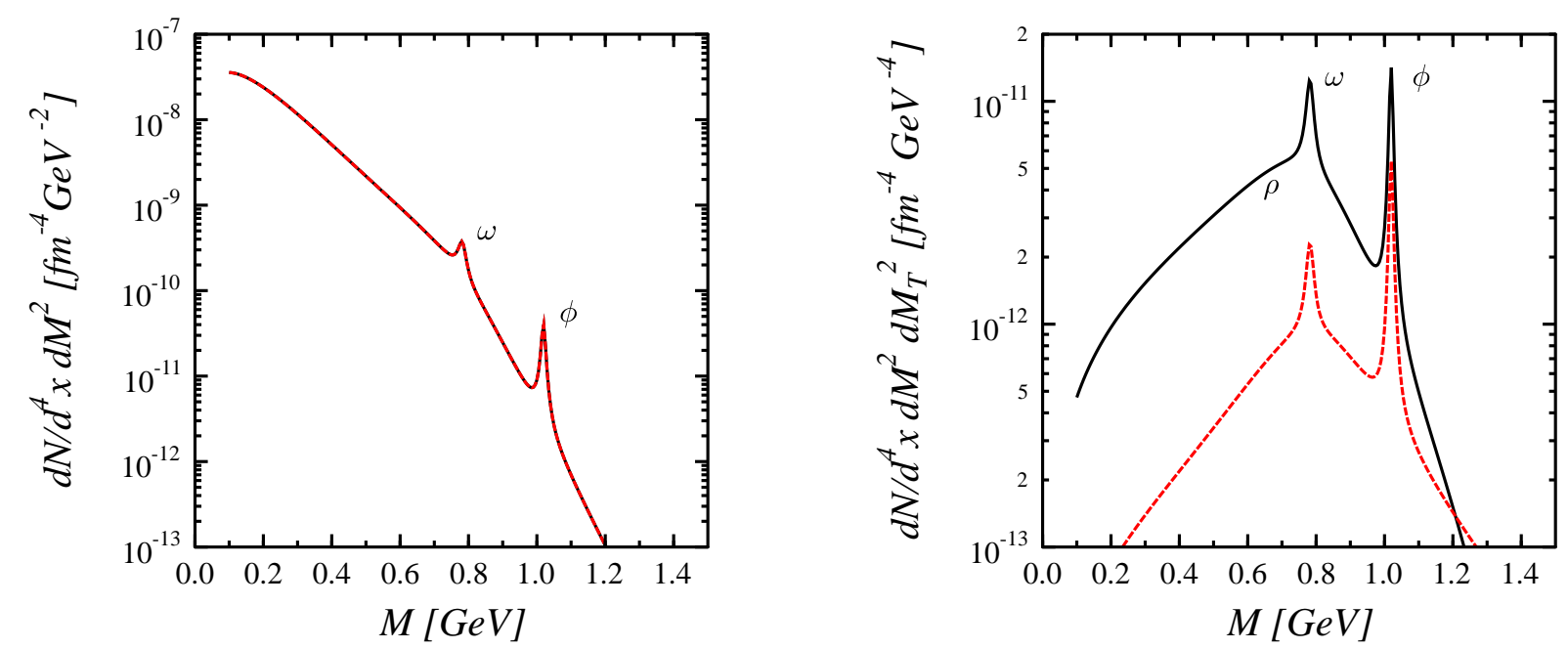

FIG. 1. The rates $d N /\left(d^{4} x d M^{2}\right)$ ((a) left panel) and $d N /\left(d^{4} x d M_{\perp}^{2} d M^{2}\right)$ ((b) right panel, $\left.M_{\perp}=1.3 \mathrm{GeV}\right)$ for $T=70 \mathrm{MeV}, v_{r}=0.1$ and 0.3 , and $b=3$.
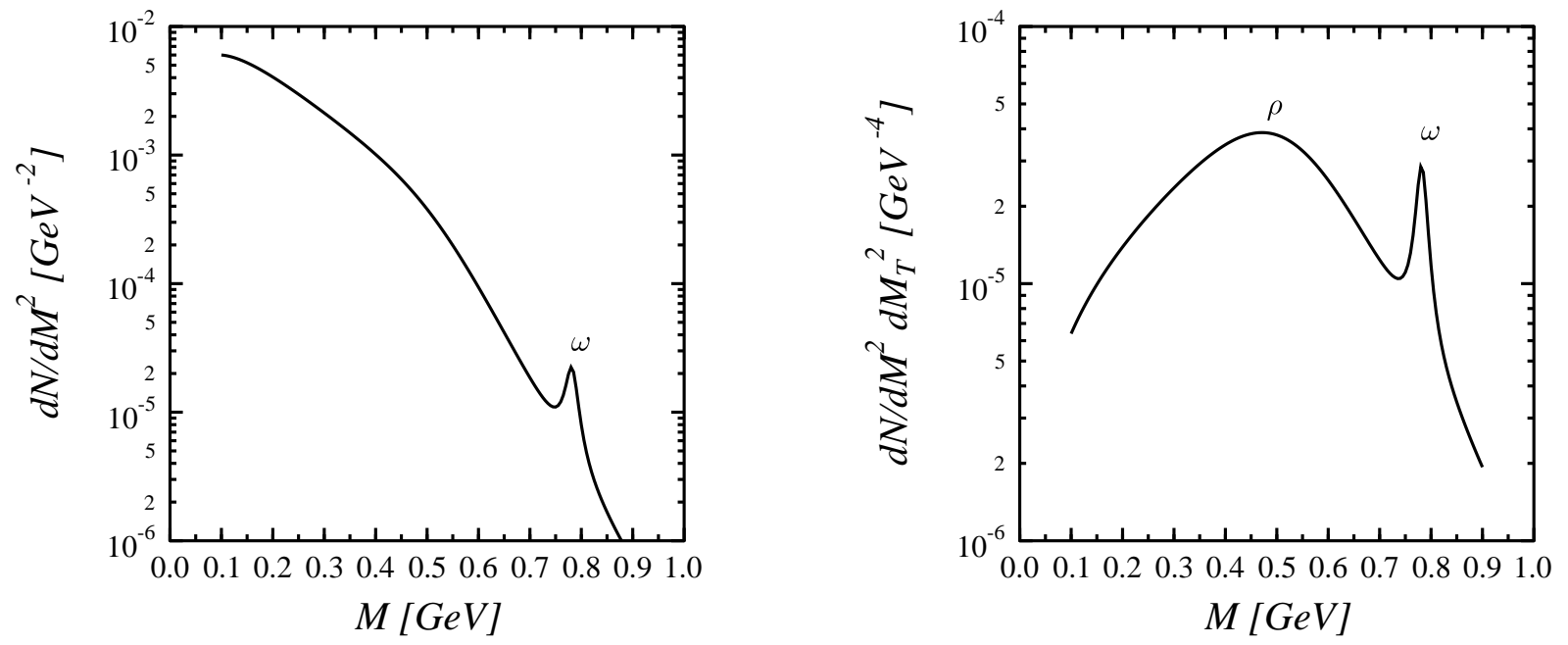

FIG. 2. The rates $d N /\left(d M^{2}\right)$ ((a) left panel) and $d N /\left(d M_{\perp}^{2} d M^{2}\right)\left((\mathrm{b})\right.$ right panel, $M_{\perp}=0.9$ $\mathrm{GeV}$ ) for the dynamical scenario described in the text and for $b=3, \Delta m_{\rho}=300 \mathrm{MeV}, \Delta m_{\omega}=0$. 

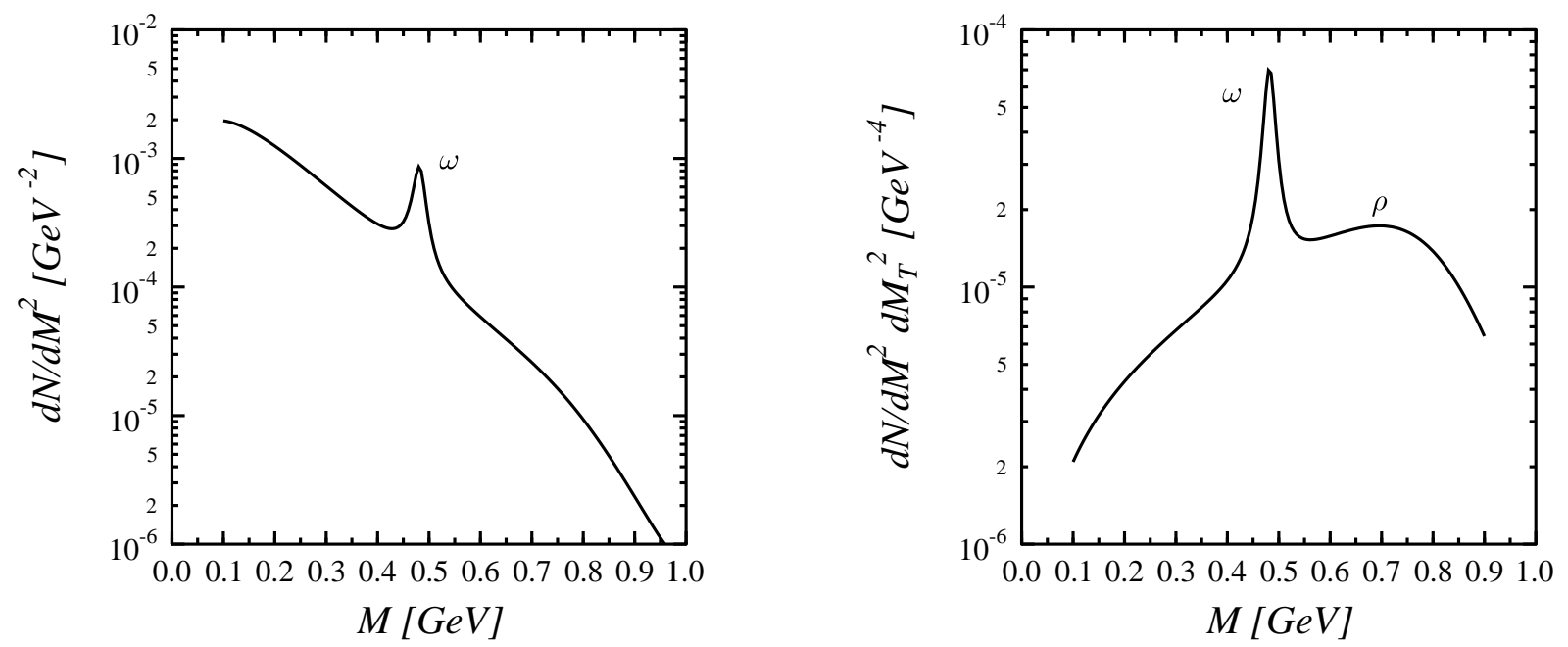

FIG. 3. As in Fig. 2 but for $\Delta m_{\rho}=0, \Delta m_{\omega}=300 \mathrm{MeV}$.
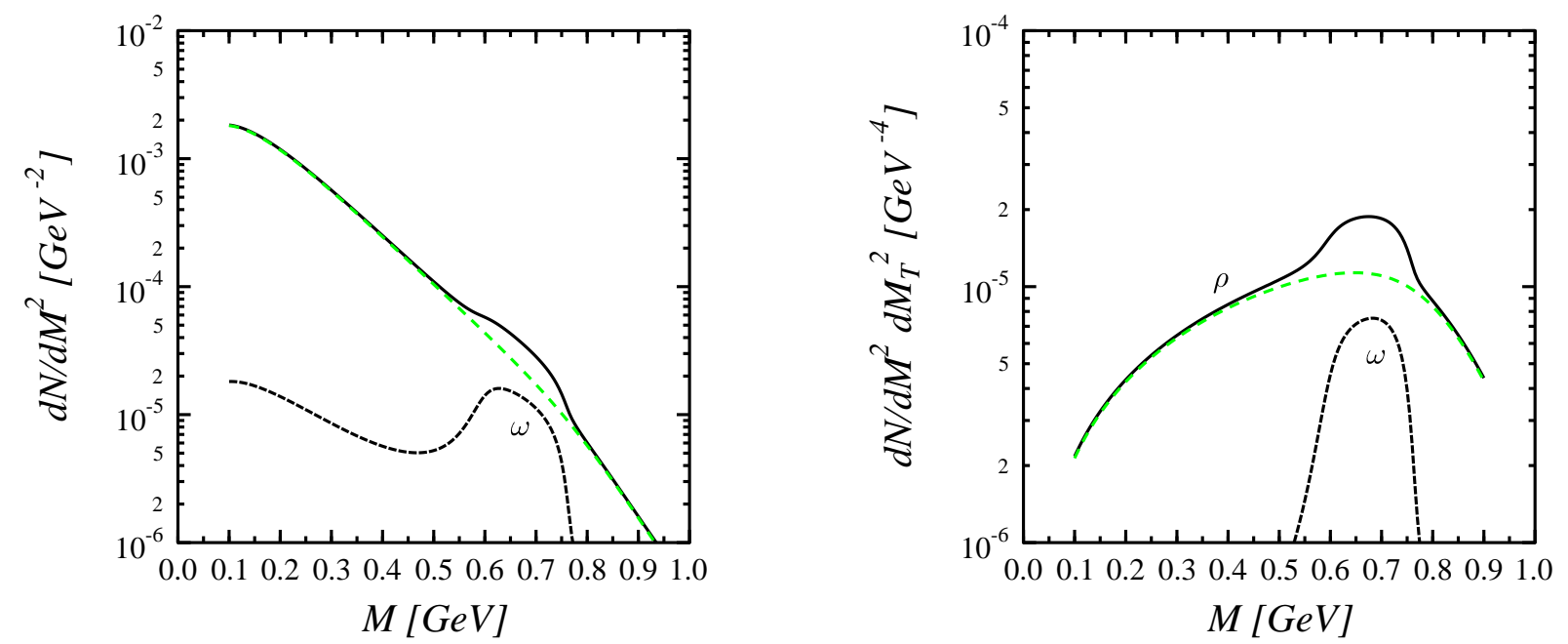

FIG. 4. As in Fig. 目 but for $\delta_{\omega}=0.08, \delta \Gamma_{\omega}=20 \mathrm{MeV}, \delta_{\rho}=0, \delta \Gamma_{\rho}=400 \mathrm{MeV}$. The $\omega(\rho)$ contribution is displayed separately as short-dashed (long-dashed) line. 